\title{
VARIACIÓN ESPACIAL EN COMUNIDADES DE ESPONJAS DE LA COSTA ORIENTAL DE BAHÍA DE COCHINOS, CUBA
}

\author{
Hansel Caballero $^{1 *}$, Linnet Busutil ${ }^{2}$, Yanel García y Pedro M. Alcolado ${ }^{3}$
}

\begin{abstract}
RESUMEN
En octubre del 2002 se estudiaron las comunidades de esponjas en cuatro localidades del arrecife de la costa este de Bahía de Cochinos, Cuba. En cada localidad se establecieron sitios de muestreo de esponjas en las zonas ecológicas de cabezos coralinos ( $3 \mathrm{~m})$, terraza somera $(6-10 \mathrm{~m})$ y terraza profunda $(15 \mathrm{~m})$. Se utilizó el método de transecto lineal de $10 \mathrm{~m}$ como unidad de muestreo para medir el cubrimiento del fondo y el marco cuadrado de $1 \mathrm{~m}$ para estimar la densidad de la comunidad. Los índices de heterogeneidad y de riqueza de especies mostraron variabilidad entre sitios y cierta tendencia a incrementarse con la profundidad. Se encontraron 30 especies pertenecientes a 19 familias y 21 géneros. Las especies más comunes fueron: Mycale laevis, Aplysina cauliformis, Cliona aprica, Aplysina fistularis y Ectyoplasia ferox, las cuales conformaron el $48 \%$ del total de individuos identificados. La densidad fue moderada (2-8 ejemplares $\left./ \mathrm{m}^{2}\right)$ y se encontró variación entre sitios. La estación más cercana a mar abierto mostró un valor inferior, posiblemente por ser la más dañada tras el paso del huracán en el 2001. El estado general de la comunidad fue bueno, según los valores de diversidad alcanzados y las especies predominantes, aunque se muestran evidencias de afectaciones recientes producto del huracán.
\end{abstract}

Palabras claves: Esponjas, comunidades, arrecifes coralinos, Cuba.

\begin{abstract}
The sponge communities at four locations on the east coast of Bahia de Cochinos were surveyed in October 2002. At each location, sampling sites were established in the ecological zones of patch reef $(3 \mathrm{~m})$, shallow terrace $(6-10 \mathrm{~m})$, and deep terrace $(15 \mathrm{~m})$. The line-transect method with a $10 \mathrm{~m}$ line as sampling unit was used to measure sponge bottom coverage, and a $1 \mathrm{~m}$ square grid was used to quantify sponge community density. Species heterogeneity and species richness indices showed variability among sites and tended to increase with depth. Thirty species belonging to 19 families and 21 genera were found. The most common species were: Mycale laevis, Aplysina cauliformis, Cliona aprica, Aplysina fistularis, and Ectyoplasia ferox, which comprised $48 \%$ of the total individuals identified. Sponge density was moderate (2-8 specimens $\left./ \mathrm{m}^{2}\right)$ and showed variation among sites. The nearest station to open sea showed a lower density value, possibly due to disturbance from the passage of a hurricane in 2001 . The general condition of the community was good, based on the diversity values reached and the predominant species, although evidence of impacts from recent hurricane were observed.
\end{abstract}

Keywords: Sponges, communities, coral reefs, Cuba.

1 Acuario Nacional de Cuba (ANC). Ave. 1ra., No. 4608, entre 46 y 60, Miramar, Playa, Ciudad de La Habana, Cuba. E-mail: hanselc@acuarionacional.cu*

2 Instituto de Oceanología (IdO). Ave. 1ra., No. 18406, entre 184 y 186, Flores, Playa, Ciudad de La Habana, Cuba. E-mail: linnet@oceano.inf.cu

Recibido 12-II-2009

Aceptado 23-IX-2009

DOI: http://dx.doi.org/10.15359/revmar.1.5

Rev. Mar. y Cost. ISSN 1659-455X. Vol. 1. 95-109, Diciembre 2009. 


\section{INTRODUCCIÓN}

Las esponjas son un componente estructural y funcional significativo de los arrecifes coralinos. Este grupo bentónico presenta elevados valores de diversidad y abundancia de especies, alta productividad, participa en el ciclo de nutrientes y tiende a ocupar los primeros lugares en biomasa en la mayoría de los hábitats marinos (Bergquist, 1978; Zea, 2001; Díaz \& Rützler, 2001).

Alcolado (2002), en su Catálogo de las esponjas de Cuba, analiza el estado en que se encuentra el conocimiento taxonómico del grupo. Considera que se halla moderadamente inventariado, e identifica al extremo más oriental de Cuba como una zona aún sin explorar. Este autor plantea, que según la literatura son reconocidas como válidas alrededor de 500 especies de esponjas y es posible que en el Gran Caribe existan más de 700 especies, sin embargo, dicho catálogo sólo compila las 280 especies que han sido registradas o recolectadas en aguas cubanas.

Sobre la ecología de las esponjas en Cuba, los estudios han estado dirigidos hacia la estructura de sus comunidades en diferentes zonas de la plataforma (Alcolado, 1979, 1985, 1989, 1992a), y han sido incluidas en evaluaciones generales del bentos (Gómez et al. 1980; De la Guardia y González-Sansón, 1997a, b y c; De la Guardia et al. 2001; De la Guardia y González, 2002; De la Guardia et al. 2003; De la Guardia et al. 2004; Valdivia y De la Guardia, 2006; entre otros).

Dada su condición de animales sésiles y longevos, resultan altamente influenciables por los factores abióticos que rigen el medio marino, por lo que han sido ampliamente utilizados como bioindicadores ambientales, tanto en Cuba como en otras regiones del mundo (Alcolado, 1984, 1990, 1992b, 1994; Alcolado y Herrera-Moreno, 1987; Muricy et al. 1991; Carballo et al. 1994; Carballo et al. 1996; entre otros). Además, se conoce de la interacción determinante de algunas especies de esponjas, para el desarrollo de otras comunidades bentónicas (Buznego y Alcolado, 1987).

Entre los años 2002 y 2003, el Acuario Nacional de Cuba realizó una evaluación de las principales comunidades arrecifales en la costa oriental de la Bahía de Cochinos, Cuba (Caballero et al. 2004a). De este estudio ya han sido publicados los resultados sobre comunidades de corales pétreos (Caballero et al. 2004b), octocorales (Varona et al. 2004) y peces (Chevalier y Cárdenas, 2005a y b).

En el presente estudio se analiza la estructura y composición de las comunidades de esponjas que conforman el arrecife que se extiende a lo largo de $30 \mathrm{~km}$ de la costa este de la Bahía de Cochinos. La información obtenida es pionera y puede servir como línea de base comparativa para futuros monitoreos de la zona, así como para lograr un mejor entendimiento de las condiciones propias de esta región en 
correspondencia con las variaciones o cambios climáticos actuales y sus afectaciones a los arrecifes coralinos.

\section{MATERIALES Y MÉTODOS}

\section{Zona de estudio}

La investigación se llevó a cabo en la costa este de la Bahía de Cochinos, al sur de la provincia de Matanzas, Cuba (Figura 1). La zona se encuentra protegida del oleaje inducido por los vientos alisios, los invernales del norte y los llamados "sures". La acción de las mareas y corrientes de marea no es significativa en esta localidad (Díaz, 1988). Sin embargo, en noviembre del 2001, el área fue azotada por el huracán Michelle, con vientos máximos sostenidos de 115 $\mathrm{km} / \mathrm{h}$ (http://.n_oaa.gov/hurricanes/ viewer.html), lo que provocó oscilaciones del nivel del mar de entre 1.5 y $3 \mathrm{~m}$, y penetraciones en la costa (observación personal). En la bahía

Figura 1

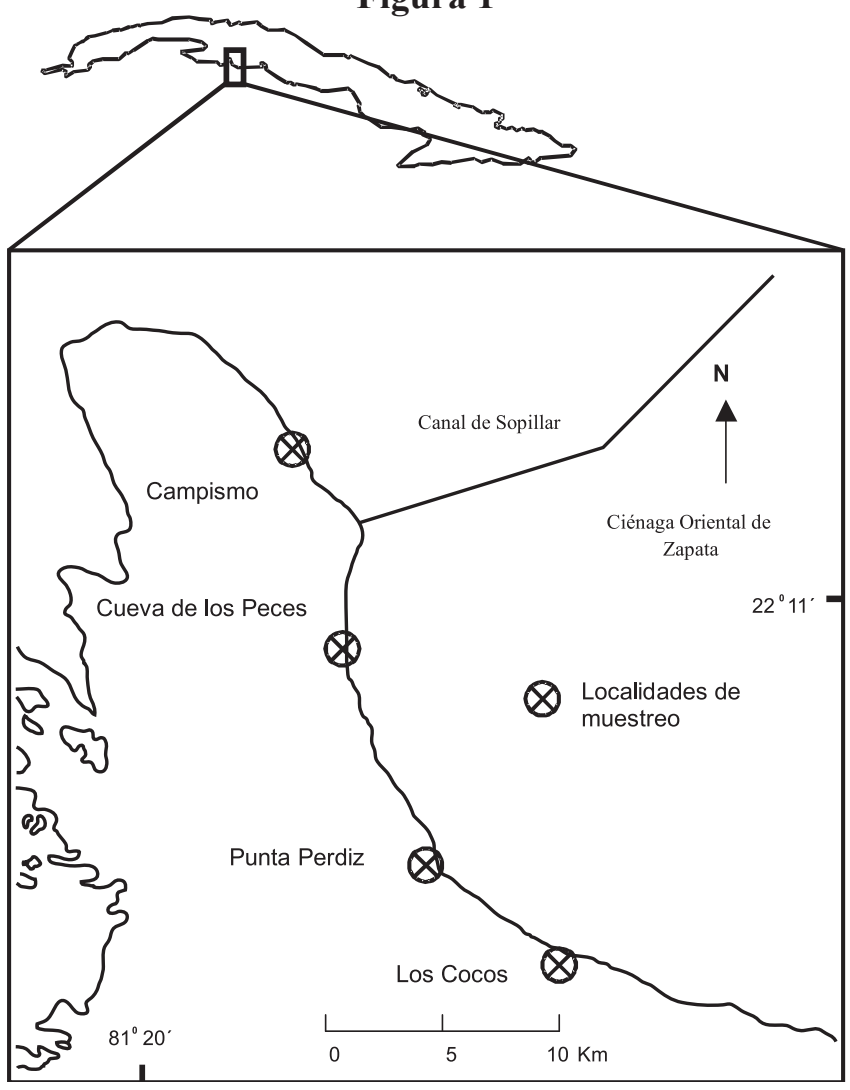

Ubicación geográfica de las localidades dentro de la zona de estudio. Location of sampling sites within the study area. 
no existen vertimientos industriales ni albañales domésticos de interés, lo que la ubica entre las menos contaminadas del país.

El arrecife se clasifica como costero, y se extiende de forma continua por más de $30 \mathrm{~km}$ bordeando la parte este de la bahía. Se establecieron cuatro localidades de muestreo a lo largo del litoral, separadas entre sí a una distancia aproximada de 5 a $9 \mathrm{~km}$. En cada localidad se establecieron tres sitios de muestreo a lo largo de un perfil perpendicular de la costa, atendiendo a las características topográficas del fondo (Cuadro 1, Figura 1).
La descripción de las zonas ecológicas (Figura 2), en orden de profundidad es la siguiente:

Cabezos (1): Extensiones rocoso-arenosas donde se despliegan promontorios de corales que varían desde pequeños cabezos hasta parches que alcanzan más de $10 \mathrm{~m}$ de diámetro y 2.5 $\mathrm{m}$ de altura. Desarrollo sésil abundante.

Terraza somera (2): Es una elevación a manera de muro o quicio de más de un metro que aparece de pronto justo antes de la pendiente del veril. Está antecedido por un corredor o canal arenoso transverso (paralelo al borde de la plataforma) que

\section{Cuadro 1}

Localidades, sitios de muestreo, profundidades de trabajo y localización geográfica.

\section{Table 1}

Location and depth of sampling sites.

\begin{tabular}{|c|c|c|c|c|}
\hline Localidad & $\begin{array}{c}\text { Localización } \\
\text { geográfica }\end{array}$ & Zona ecológica & Sitio & Profundidad (m) \\
\hline \multirow{3}{*}{ Campismo } & \multirow{3}{*}{$\begin{array}{l}22^{\circ} 15^{\prime} \mathrm{LN} \\
81^{\circ} 10^{\prime} \mathrm{LW}\end{array}$} & cabezos & CA1 & 3 \\
\hline & & terraza somera & CA2 & 6 \\
\hline & & terraza profunda & CA3 & 15 \\
\hline \multirow{3}{*}{ Cueva de los Peces } & \multirow{3}{*}{$\begin{array}{l}22^{\circ} 11^{\prime} \mathrm{LN} \\
81^{\circ} 09^{\prime} \mathrm{LW}\end{array}$} & cabezos & $\mathrm{CP} 1$ & 3 \\
\hline & & terraza somera & $\mathrm{CP} 2$ & 6 \\
\hline & & terraza profunda & $\mathrm{CP} 3$ & 15 \\
\hline \multirow{3}{*}{ Punta Perdiz } & \multirow{3}{*}{$\begin{array}{l}22^{\circ} 07^{\prime} \mathrm{LN} \\
81^{\circ} 08^{\prime} \mathrm{LW}\end{array}$} & cabezos & PP1 & 3 \\
\hline & & terraza somera & PP2 & 5 \\
\hline & & terraza profunda & PP3 & 15 \\
\hline \multirow{3}{*}{ Los Cocos } & \multirow{3}{*}{$\begin{array}{l}22^{\circ} 05^{\prime} \mathrm{LN} \\
81^{\circ} 05^{\prime} \mathrm{LW}\end{array}$} & cabezos & LC1 & 6 \\
\hline & & terraza somera & $\mathrm{LC} 2$ & 10 \\
\hline & & terraza profunda & LC3 & 15 \\
\hline
\end{tabular}


Figura 2

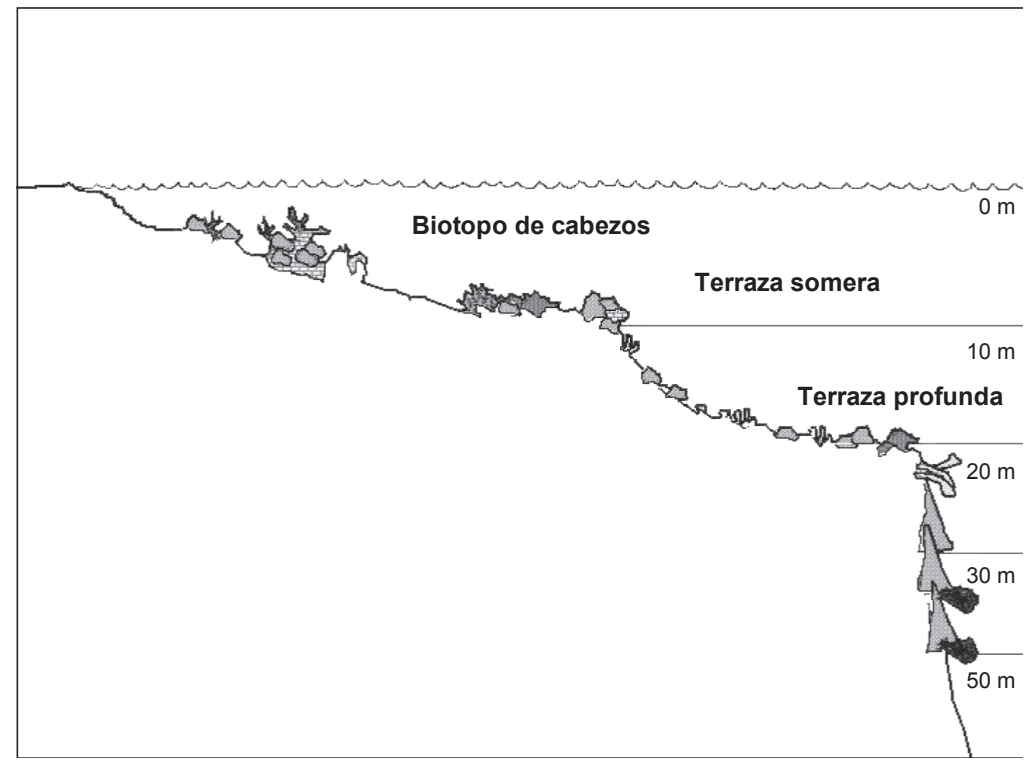

Representación esquemática del perfil de fondo del arrecife. Se presentan las zonas ecológicas donde se situaron los sitios de muestreo.

Schematic representation of the reef bottom profile. The ecological zones were samplings were carried out are shown.

lo delimita de la zona coralina menos profunda adyacente. Presenta varios parches de arena. Desarrollo sésil abundante.

Terraza profunda (3): Pendiente irregular con inclinación variable de $45^{\circ}$ a $60^{\circ}$ hasta los $20 \mathrm{~m}$ donde el talud cae abruptamente. Presenta canales de arena perpendiculares al borde del arrecife. Desarrollo abundante de corales y esponjas.

\section{Metodología de muestreo y proce- samiento de datos}

La comunidad se muestreó en octubre del 2002 mediante buceo autónomo. Los ejemplares se identi- ficaron in situ, según la terminología del Catálogo de las esponjas de Cuba de Alcolado (2002).

Se determinó la diversidad y composición por especies. Se utilizaron como índices de diversidad: el número de especies (S), la heterogeneidad de Shannon y Weaver (1963) y la equitatividad de Pielou (1966).

El porcentaje de cubrimiento del fondo por esponjas se evaluó mediante el método de transecto lineal de Loya (1978) de $10 \mathrm{~m}$, para lo que se emplearon 10 transectos por sitio.

Para estimar la densidad de esponjas se utilizó el marco cuadrado de $1 \mathrm{~m}$ de lado como unidad de muestreo. Se muestrearon de 30 a 43 
marcos, colocados al azar en cada sitio, en dependencia de la cantidad requerida para obtener curvas con tendencia asintótica de número de especies contra número de individuos acumulados.

Se realizó un análisis de varianza anidada (ANOVA, localidades-sitios) para conocer la existencia de diferencias significativas en el cubrimiento y la densidad de esponjas. Se comprobó la homogeneidad de varianza de los datos mediante un análisis de la relación de Taylor (1961), según la metodología de Elliot (1971). Para determinar las diferencias entre los pares de medias se utilizó la prueba de Student-Newman-Keuls (SNK), con nivel de significación de 0.05 .

Los cálculos se realizaron ejecutando los programas PRIMER 5 y STATISTICA 6.0 para computadoras compatibles con IBM.

\section{RESULTADOS}

En total se encontraron 30 especies de esponjas pertenecientes a 19 familias y 21 géneros. Las más comunes fueron: Mycale laevis, Aplysina cauliformis, Cliona aprica, Aplysina fistularis y Ectyoplasia feroz, las cuales conformaron el $48 \%$ del total de individuos identificados (Cuadro 2). En los sitios más someros dominó M. laveis, seguidas por C. aprica y A. cauliformis. A mayor profundidad se encontró variabilidad en la dominancia de especies. En el Campismo dominó Ircinia felix; en Cueva de los Peces, Neofibularia nolitangere, Iotrochota birotulata y E. ferox; en Punta Perdiz, A. cauliformis y E. ferox, mientras que en Los Cocos las más abundantes fueron: Spirastrella coccinea y M. laevis.

El índice de heterogeneidad varió entre 2,1 y 2,7 natios con cierta tendencia a incrementarse con la profundidad. La riqueza de especies varió entre 10 y 22 especies por sitio y mostró un patrón similar a la heterogeneidad. La equitatividad fue muy alta y muy parecida en todo el arrecife, con valores entre 0.87 y 0.93 (Cuadro 2, Figura 3).

El cubrimiento de esponjas fue bajo $(<6 \%)$ y no se encontraron diferencias entre los sitios, a excepción de CP3 que presentó un valor significativamente mayor (8\%).

Se encontró interacción significativa localidades-sitios para la densidad de esponjas. El análisis de SNK de los promedios de densidad mostró tres grupos de sitios con valores significativamente distintos, donde los menores se presentaron en Los Cocos, y el mayor en la terraza profunda de Cueva de los Peces (Figura 4).

\section{DISCUSIÓN}

Siguiendo los criterios de Alcolado (1992a), los índices de heterogeneidad y de equitatividad encontrados en Bahía de Cochinos indican que la zona de forma general es muy constante (a juzgar por J') y presenta condiciones favorables (a juzgar por 


\section{Cuadro 2}

Abundancia (\%) de esponjas (se presenta el 95\% del total de especies identificadas) y riqueza de especies ( $\mathrm{S}$ ). $\mathrm{N}=$ número total de esponjas, $\mathrm{UM}=$ número de unidades de muestreo. Ver código de los sitios en el Cuadro 1.

\section{Table 2}

Sponge abundance (\%) for $95 \%$ of the total species identified, and species richness $(\mathrm{S}) . \mathrm{N}=$ total number of sponges, $\mathrm{UM}=$ number of sampling units. Legend same as in Table 1.

\begin{tabular}{|c|c|c|c|c|c|c|c|c|c|c|c|c|}
\hline Especies & CA1 & CP1 & PP1 & LC1 & CA2 & CP2 & PP2 & LC2 & CA3 & CP3 & PP3 & LC3 \\
\hline Mycale laevis & 21.5 & 20.8 & 9.6 & 18.6 & 18.8 & 8.4 & 8.4 & 18.6 & 2.9 & 6.6 & 7.3 & 15.5 \\
\hline $\begin{array}{l}\text { Aplysina } \\
\text { cauliformis }\end{array}$ & 4.2 & 6.5 & 5.4 & 9.3 & 10.7 & 7.1 & 11.2 & 14.4 & 11.8 & 7.4 & 16.6 & 10.0 \\
\hline Cliona aprica & 19.4 & 20.8 & 14.4 & 15.1 & 20.1 & 9.0 & 6.4 & 5.9 & 2.4 & 1.6 & 1.6 & 8.2 \\
\hline Aplysina fistularis & 9.7 & 8.4 & 3.0 & 20.9 & 10.1 & 7.1 & 11.2 & 9.3 & 12.9 & 7.0 & 6.2 & 7.3 \\
\hline Ectyoplasia ferox & 8.3 & 15.6 & 4.8 & 0.0 & 14.8 & 8.4 & 0.0 & 4.2 & 7.1 & 11.1 & 14.5 & 6.4 \\
\hline $\begin{array}{l}\text { Iotrochota } \\
\text { birotulata }\end{array}$ & 0.0 & 0.0 & 16.2 & 8.1 & 0.0 & 0.0 & 21.7 & 0.0 & 12.9 & 11.5 & 1.0 & 0.0 \\
\hline Ircinia felix & 5.6 & 3.2 & 0.0 & 4.7 & 10.1 & 11.0 & 5.2 & 9.3 & 17.6 & 9.8 & 0.0 & 5.5 \\
\hline $\begin{array}{l}\text { Smenospongia } \\
\text { aurea }\end{array}$ & 1.4 & 11.0 & 13.8 & 0.0 & 2.7 & 11.6 & 12.0 & 0.0 & 1.8 & 3.3 & 10.9 & 0.0 \\
\hline $\begin{array}{l}\text { Scopalina } \\
\text { ruetzleri }\end{array}$ & 14.6 & 6.5 & 3.0 & 0.0 & 0.0 & 3.2 & 13.7 & 11.9 & 0.0 & 2.5 & 5.7 & 10.0 \\
\hline $\begin{array}{l}\text { Aiolochroia } \\
\text { crassa }\end{array}$ & 4.2 & 1.9 & 3.6 & 5.8 & 0.7 & 8.4 & 0.0 & 2.5 & 6.5 & 7.0 & 7.8 & 9.1 \\
\hline $\begin{array}{l}\text { Neofibularia } \\
\text { notilangere }\end{array}$ & 0.0 & 0.0 & 0.0 & 0.0 & 4.0 & 15.5 & 0.8 & 0.0 & 1.8 & 12.3 & 2.6 & 0.0 \\
\hline $\begin{array}{l}\text { Spirastrella } \\
\text { coccinea }\end{array}$ & 0.0 & 0.0 & 8.4 & 0.0 & 0.0 & 0.0 & 6.8 & 11.0 & 1.2 & 0.0 & 1.6 & 16.4 \\
\hline Mycale laxissima & 0.0 & 0.0 & 0.0 & 0.0 & 4.0 & 2.6 & 0.0 & 0.0 & 2.4 & 5.7 & 6.7 & 1.8 \\
\hline Cliona delitrix & 2.1 & 5.2 & 2.4 & 7.0 & 0.0 & 1.3 & 0.0 & 0.0 & 3.5 & 1.6 & 4.1 & 0.0 \\
\hline Niphates digitales & 2.1 & 0.0 & 0.0 & 3.5 & 1.3 & 1.3 & 1.2 & 4.2 & 1.2 & 2.0 & 2.6 & 5.5 \\
\hline Cliona varians & 1.4 & 0.0 & 2.4 & 0.0 & 0.0 & 2.6 & 0.0 & 0.0 & 0.6 & 2.5 & 1.6 & 0.0 \\
\hline Cliona sp. & 0.0 & 0.0 & 8.4 & 0.0 & 0.0 & 0.0 & 0.0 & 0.0 & 0.0 & 0.0 & 3.1 & 0.0 \\
\hline $\begin{array}{l}\text { Plakortis } \\
\text { angulospiculatus }\end{array}$ & 0.0 & 0.0 & 0.0 & 0.0 & 0.0 & 0.0 & 0.0 & 2.5 & 3.5 & 0.4 & 1.6 & 2.7 \\
\hline $\mathrm{S}$ & 13 & 10 & 17 & 11 & 12 & 16 & 12 & 14 & 20 & 22 & 22 & 13 \\
\hline UM & 30 & 30 & 36 & 30 & 31 & 30 & 43 & 32 & 30 & 30 & 36 & 30 \\
\hline $\mathrm{N}$ & 144 & 154 & 167 & 86 & 149 & 155 & 249 & 118 & 170 & 244 & 193 & 110 \\
\hline
\end{tabular}


Figura 3

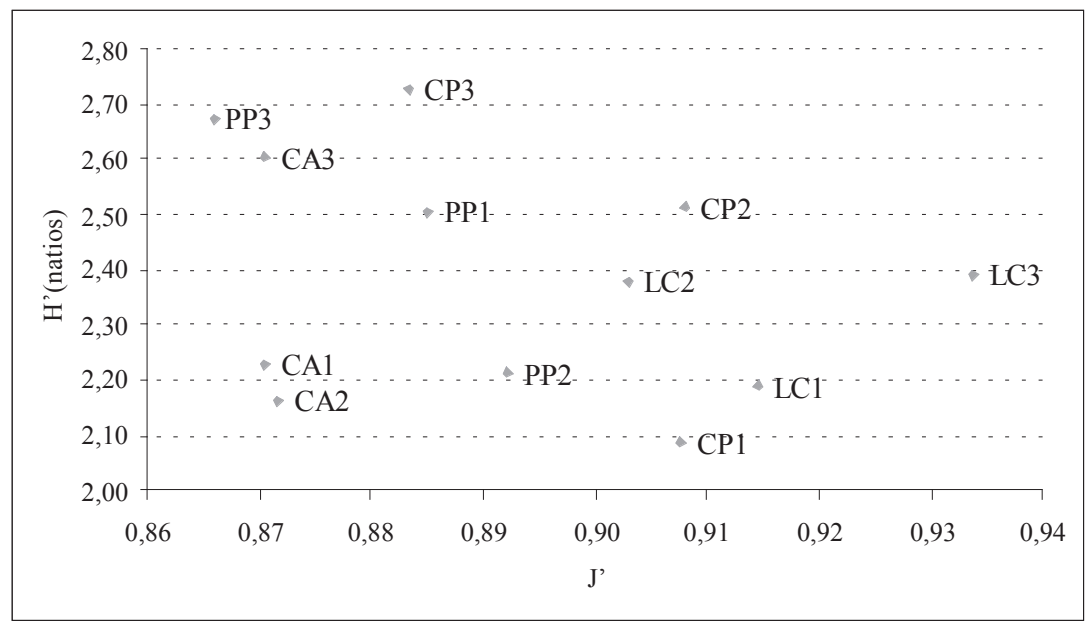

Gráfico de dispersión de los sitios de muestreo en relación con el índice de heterogeneidad ( $\left.\mathrm{H}^{\prime}\right)$ y el de equitatividad (J'). Ver código de los sitios en el Cuadro 1.

Scatter plot of the heterogeneity ( $\left.\mathrm{H}^{\prime}\right)$ versus the evennes index (J'), for the sampling sites. Legend same as in Table 1.

Figura 4

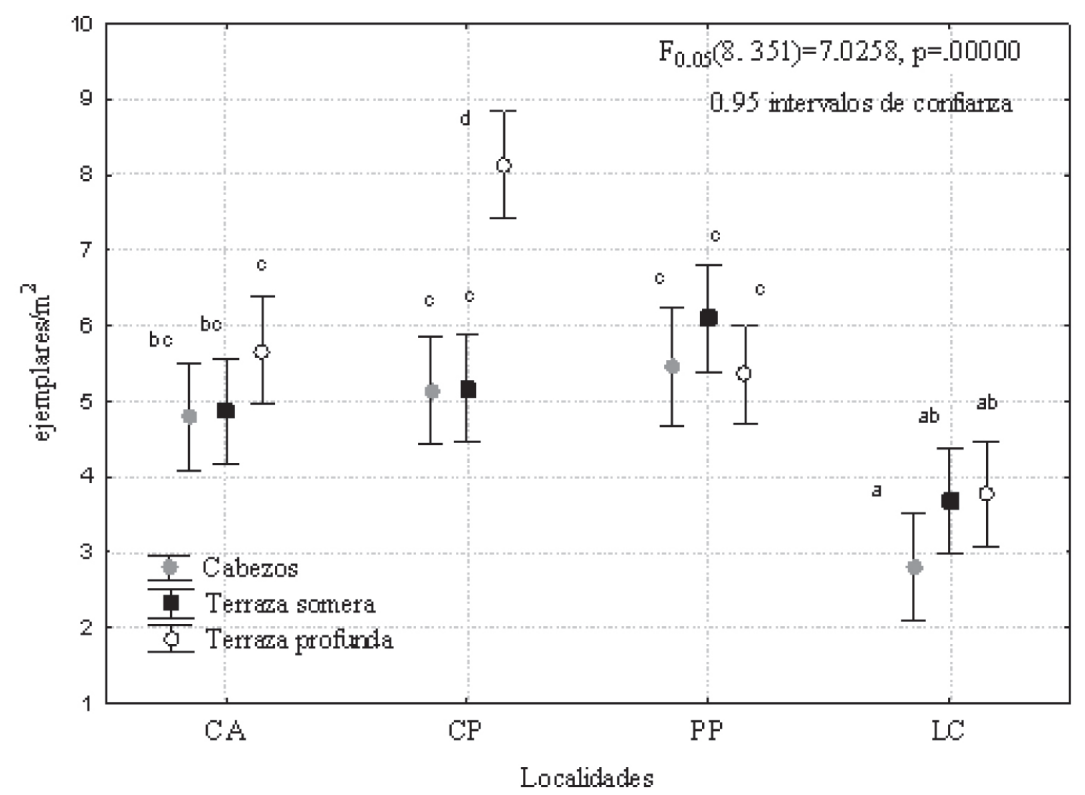

Indicadores de densidad obtenidos de las comunidades de esponjas en cada sitio de muestreo. Ver código de los sitios en el Cuadro 1.

Density indicators for the sponge communities at each sampling site. Legend same as in Table 1 . 
H') para el desarrollo de las esponjas. Este autor propone un sistema de 11 categorías basado en los valores de heterogeneidad (H') y equitatividad (J') que alcanzan las esponjas, y que permite caracterizar comparativamente al ambiente en cuanto al grado de severidad y de predictibilidad. Esta característica está dada por la elevada conexidad especie-ambiente que presentan las esponjas no crípticas, o sea, su estructura comunitaria está más determinada por los factores abióticos del entorno que por relaciones intra o interespecíficas, por lo que reflejan las condiciones ambientales imperantes (Alcolado, 1984).

Según este sistema, los índices de diversidad determinados en nuestro estudio caracterizan a los sitios de cabezos y terrazas someras como zonas constantes y algo estresadas. La tensión podría estar relacionada con el efecto restrictivo del oleaje sobre estas comunidades. A pesar de esto, los índices de diversidad encontrados en Bahía de Cochinos se pueden considerar altos al compararlos con los obtenidos a igual profundidad en arrecifes del norte de La Habana (Alcolado, 1989; De la Guardia y González-Sansón, 1997c; González, 1999); arrecifes que, dada su posición geográfica, enfrentan un oleaje más intenso durante el año, producto del predominio en la zona de los vientos del norte y del nordeste, sobre todo durante los frentes fríos. Sólo la terraza somera de Cueva de los Peces en Bahía de Cochinos su- giere la presencia de condiciones más favorables y constantes, dado que su fondo se distingue por un relieve más acentuado, con zonas más resguardadas y expuestas en grado variable a la radiación solar. Estas condiciones determinan en parte los altos valores de H' y J' encontrados en esta estación, e influyen en el comportamiento de otras variables que se discuten más adelante. De por sí, la posición a sotavento de todos los sitios ya tiene implicaciones en que los índices H' y $\mathrm{J}$ ' sean elevados, independientemente de las particularidades de cada sitio.

Las especies dominantes en los sitios someros corroboran la caracterización antes expuesta. La esponja Mycale laevis frecuentemente se asocia con el coral Montastraea annularis. Esta relación mutualista ha sido descrita por Goreau y Hartman (1996) como indicadora de un buen estado de salud en arrecifes coralinos, dado que resulta el ambiente beneficioso para ambas especies y favorece el desarrollo de las estructuras arrecifales. Dicha situación parece determinar en nuestro caso que $M$. laevis es la especie más común, siendo el coral igual de dominante en la zona, según Caballero et al. (2004b). Dichos autores también refieren que no existen diferencias significativas en cuanto a la densidad de corales entre los dos biotopos someros, situación también observada en las comunidades de esponjas (exceptuando el sitio Los Cocos). Por su parte, De la Guardia y González-Sansón (1997a) 
refieren que $M$. laevis es común en zonas con alto cubrimiento coralino, característica que cumplen nuestros sitios de estudio.

Le siguen como especies dominantes en los cabezos y terrazas someras: Cliona aprica y Aplysina cauliformis. La primera suele ser dominante cuando existe una fuerte agitación del agua, en zonas sometidas a embate constante y esporádicamente afectadas por ciclones (Alcolado, 1989, 1992a, 1999; De la Guardia y González-Sansón, 1997a). Alcolado (trabajo en preparación) ha observado que $A$. cauliformis se ha destacado en sitios afectados con cierta frecuencia por ciclones, como el este y el sur del Golfo de Batabanó. La segunda especie ha sido reportada como indicadora de sedimentación y agitación moderada (Alcolado y Herrera-Moreno, 1987), condiciones ambas que se presentan en la zona.

De acuerdo con H'y J', las terrazas profundas indican ser favorables y constantes. Únicamente la terraza profunda de Los Cocos se excluye de esta categoría, y se caracteriza por sugerir un ambiente constante y algo estresado. En este último sitio, se aprecia, además, un número de especies considerablemente menor que en los otros sitios con este biotopo. Es posible que el paso del huracán Michelle por la zona en el 2001 sea la causa sustancial de ello. Este sitio se encuentra menos protegido geográficamente y, por lo tanto, debió sufrir con mayor intensidad su influencia.
A mayor profundidad se observaron mayores diferencias entre estaciones, que pueden estar dadas por las condiciones ambientales propias de cada sitio, o por alguna componente estocástica, como es de esperar en ambientes no sometidos a factores determinísticos fuertemente selectivos como el oleaje, la sedimentación y la luz intensa (Alcolado, 1998).

Existen coincidencias entre las especies que fueron dominantes en Cueva de los Peces (Iotrochota birotulata y Ectyoplasia ferox), Punta Perdiz (E. ferox) y Los Cocos (Mycale laevis), con las reportadas por Alcolado (1989) para el arrecife de Rincón de Guanabo, no siendo igual en el resto de los sitios, donde se desconocen estudios que corroboren al resto de las especies más abundantes (Neofibularia nolitangere, Ircinia felix y Spirastrella coccinea) como típicas de ambientes más profundos. Sobre la especie I. birotulata en particular, Alcolado (1979) y De la Guardia y González-Sansón (1997a) también encontraron las mayores abundancias en las zonas profundas.

El área de estudio se caracteriza como limpia, lo que pone en evidencia la especie Aplysina fistularis, típica de estos ambientes, según Alcolado y Herrera-Moreno (1987), y que presentó valores de abundancia entre moderados y altos en todos los sitios (Cuadro 2). Esta alcanzó su valor máximo en la estación LC1, que es la más próxima a mar abierto y por consiguiente con mayor influencia de 
la circulación marina. Esta especie, además, ha sido reportada en lugares sometidos a la resuspensión y abrasión de sedimentos arenosos (Alcolado y Herrera-Moreno, 1987), condiciones también presentes en grado variable entre los sitios muestreados.

Los bajos porcentajes de cubrimiento en todas los sitios nos hacen suponer que los arrecifes de esta zona se encuentran en su estado natural donde predominan los corales, lo que es coincidente con los muestreos de Caballero et al. (2004b). De la Guardia y González-Sansón (1997b) obtuvieron resultados similares en un arrecife de la costa noroccidental de Cuba.

El cubrimiento de esponjas en el sitio CP3 mostró diferencias significativas con respecto al resto, pero aún así el valor fue bajo. Como se explicó anteriormente, el fondo de este sitio presenta un relieve marcado que favorece el desarrollo de las esponjas, lo que puede explicar esas diferencias.

La densidad de esponjas fue moderada en la mayoría de los sitios. La menor densidad de Los Cocos puede ser atribuida a la posible acción destructiva del huracán Michelle en el 2001. A pesar de no existir datos históricos para comparar, sí se tienen criterios anecdóticos de los pobladores de la zona y nuestros, sobre la existencia en este sitio de un mayor número de esponjas grandes antes del paso de ese huracán.

Investigaciones en arrecifes del Gran Caribe que han sufrido el paso de ciclones de gran intensidad han descrito cambios de importancia en la biomasa y densidad de comunidades de esponjas, especialmente en las especies blandas y en las digitadas erectas que se rompen desde la base (Salazar-Vallejo, 2002). Se han encontrado disminuciones de hasta un $25 \%$ del índice de biomasa, pérdidas de más de 2 ejemplares $/ \mathrm{m}^{2}$ y alteraciones en el patrón de dominancia de las especies (Wulff, 1995; Bahena et al. 2000, citados por Salazar-Vallejo, 2002). Estos autores encontraron los cambios más llamativos en profundidades entre 5 y $9 \mathrm{~m}$.

Los sitios someros presentaron densidades parecidas a las reportadas por Alcolado (1989) para Rincón de Guanabo, inferiores a las de Playa Baracoa (Alcolado, 1990) y superiores a las de barlovento (Alcolado, 1979). En los sitios de terraza profunda encontramos densidades inferiores a las de otras zonas del litoral noroccidental (Alcolado, 1979, 1989, 1990). Se desconoce si estas condiciones se han mantenido en el tiempo, ya que no contamos con estudios más recientes de nuestra área.

\section{CONCLUSIONES}

Las condiciones físico-geográficas de la Bahía de Cochinos favorecen el desarrollo de las esponjas. Las especies predominantes y los valores de diversidad alcanzados denotan el buen estado de salud en que se encuentran estas comunidades, aún cuando los valores de 
densidad y cubrimiento no fueron altos debido al azote de un huracán. Las presiones ambientales, propias de los biotopos arrecifales de esta región, determinan la estructura y composición del grupo.

\section{BIBLIOGRAFÍA}

Alcolado, P. M. (1979). Estructura ecológica de la comunidad de esponjas en un perfil costero de Cuba. Cienc. Biol., 3, 105-127.

Alcolado, P. M. (1984). Nuevas especies de esponjas encontradas en Cuba. Poeyana, 271, 1-22.

Alcolado, P. M. (1985). Estructura ecológica de las comunidades de esponjas en Punta del Este, Cuba. Rep. Invest. Inst. Oceanología, 38, 1-65.

Alcolado, P. M. (1989). Estructura ecológica de las comunidades de esponjas del arrecife del Rincón de Guanabo, Cuba. Rep. Invest. Inst. Oceanología, 10, 1-16.

Alcolado, P. M. (1990). General feature of Cuban sponge communities. En: K. Rützler (ed.), New Perspectives in Sponges Biology (pp. 134-142). Washington D.C.: Smithsonian Institution Press.

Alcolado, P. M. (1992a). Comunidades de esponjas en las bahías de Jururú y Vita, Cuba. Rep. Invest. Inst. Oceanología, 10, 1-12.

Alcolado, P. M. (1992b). Sobre la interpretación del ambiente marino mediante el empleo de los índi- ces de diversidad y equitatividad. Cienc. Biol., 24, 124-127.

Alcolado, P. M. (1994). General trends in coral reef sponges communities of Cuba. En: R.W.M. van Soest, Th. M. G. van Kempien and J. C. Braekman (eds.), Sponges in time and space (pp. 225-233). Rotterdam: Balkema.

Alcolado, P. M. (1998). Controversia Determinismo-Caos. Avicennia, 8, 22-26.

Alcolado, P. M. (1999). Comunidades de esponjas de los arrecifes del Archipiélago Sabana-Camagüey. Bol. Invest. Mar. Cost., 28, 95-124.

Alcolado, P. M. (2002). Catálogo de las esponjas de Cuba. Avicennia, 15, 53-72.

Alcolado, P. M. y Herrera-Moreno, A. (1987). Efectos de la contaminación sobre las comunidades de esponjas en el litoral de La Habana, Cuba. Rep. Invest. Inst. Oceanología, 68, 1-23.

Bergquist, P. R. (1978). Sponge ecology. En Sponges. Hutchinson: Londres. 268 p.

Buznego, M.y Alcolado, P. M. (1987).

Frecuencia de ataques de esponjas perforadoras sobre algunos corales escleractinios y otros sustratos marinos en Cuba. Rep. Invest. Inst. Oceanología, 59, 1-18.

Caballero, H., Chevalier, P., Varona, G., Cárdenas, A. L., Pastor, L., Pérez-Hernández, A. y García, Y. (2004a). Componentes más comunes de la fauna del arrecife de 
coral de la costa oriental de Bahía de Cochinos, Cuba: Corales, esponjas, gorgonáceos y peces. Rev. Invest. Mar., 25 (1), 37-44.

Caballero, H., Varona, G. y García, Y. (2004b). Estructura ecológica de las comunidades de corales de la costa oriental de Bahía de Cochinos, Cuba. Rev. Invest. Mar., 25 (1), 23-36.

Carballo, J. L., Naranjo, S. A. \& García-Gómez, J. C. (1996). Use of marine sponges as stress indicators in marine ecosystems at Algeciras Bay (southern Iberian Peninsula). Mar. Ecol. Progr. Ser., 135, 109-122.

Carballo, J. L., Sánchez-Moyano, J. E. \& García-Gómez, J. C. (1994). Taxonomic and ecological remarks on boring sponges Clionidae from the Straits of Gibraltar Southern Spain: Tentative Bioindicators? Zool. J. Linnean Soc., 112, 407-424.

Chevalier, P. P. y Cárdenas, A. L. (2005a). Variación espacial y temporal de las asociaciones de peces de arrecifes costeros de la costa oriental de Bahía de Cochinos. I: Abundancia y diversidad. Rev. Invest. Mar., 26 (1), 45-57.

Chevalier, P. P. y Cárdenas, A. L. (2005b). Variación espacial y temporal de las asociaciones de peces de arrecifes costeros de la costa oriental de Bahía de Cochinos. II: Análisis multidimensional. Rev. Invest. Mar., 26 (1), 59-66.
De la Guardia, E. y González-Sansón, G. (1997a). Asociaciones de esponjas, gorgonias y corales de un arrecife en la costa noroccidental de Cuba. I: Distribución espacial de biotopos. Rev. Invest. Mar., 18 (3), 198-207.

De la Guardia, E. y González-Sansón, G. (1997b). Asociaciones de esponjas, gorgonias y corales de un arrecife en la costa noroccidental de Cuba. II: Variaciones espaciales del cubrimiento y la densidad. Rev. Invest. Mar., 18 (3), 208-215.

De la Guardia, E. y González-Sansón, G. (1997c). Asociaciones de esponjas, gorgonias y corales de un arrecife en la costa noroccidental de Cuba. III: Variación espacial de la diversidad. Rev. Invest. Mar., 18 (3), 216-222.

De la Guardia, E. y González, P. (2002). Comunidad bentónica en arrecifes coralinos de Punta del Este y Cayo Largo, Archipiélago de los Canarreos, Cuba. Rev. Invest. Mar., 23 (3), 185-194.

De la Guardia, E., González, P. y Trelles, J. (2001). Macrobentos del arrecife coralino adyacente al río Almendares, Habana, Cuba. Rev. Invest. Mar., 22 (3), 167-178.

De la Guardia, E., González, P., Varona, G., González-Ferrer, S. y Superes, W. (2003). Variaciones temporales y espaciales en la comunidad bentónica del arrecife de playa Herradura, provincia Haba- 
na, Cuba. Rev. Invest. Mar., 24 (2), 117-126.

De la Guardia, E., Valdivia, A. y González, P. (2004). Estructura de comunidades bentónicas en la zona de buceo de María la Gorda, Ensenada de Corrientes, sureste de la Península de Guanahacabibes, Cuba. Rev. Invest. Mar., 25 (2), 103-111.

Díaz, R. (1988). Derrotero de las costas de Cuba: Región Maritima del Sur. La Habana, Cuba: Editorial Científico-Técnica del Instituto Cubano del Libro. 200 p.

Díaz, M. C. \& Rützler, K. (2001). Sponges: An essential component of Caribbean Coral Reefs. Bull. Mar. Sci., 69 (2), 535-546.

Elliot, J. M. (1971). Some methods of the statistical analysis of samples of benthic invertebrates. Fresh Water Biological Association. Sci. Publ., 25, 1-144.

Gómez, O., Ibarzábal, D. y Silva, A. (1980). Evaluación cuantitativa de bentos en la región suroccidental de Cuba. Inf. Cien.-Téc., Inst. Oceanología, 149, 1-25.

González, P. (1999). Comunidades de esponjas, corales y gorgonias en un arrecife coralino costero de Ciudad de La Habana. Trabajo de Diploma. Universidad de La Habana, 25 p.

Goreau, T. F. \& Hartman, W. D. (1996). Sponge: Effect on the form of reef corals. Sci., 151 (3708), 343-344.

Loya, Y. (1978). Plotless and transect methods. En: D. R. Stoddart and R. E. Johannes (eds.), Coral reef research methods (pp: $197-$ 217). Paris: UNESCO.

Muricy, G., Hajdu, E., Custodio, M., Klautau, M., Russo, C. \& Peixinho, S. (1991). Sponge distribution at Arrial do Cabo, S. E. Brazil. Proc. VII Sym. Coast Oceanograph. Management ASCE, 2, 1138-1196.

Pielou, E. C. (1966). Shannon's formula as a measurement of specific diversity: its use and mesure. Am. Nat., 100, 463-465.

Salazar-Vallejo, S. (2002). Huracanes y biodiversidad costera tropical. Rev. Biol. Trop., 50 (2), 415 428.

Shannon, C. E. \& Weaver, W. (1963). The mathematical theory of communication. En: J. A. Ludwig (ed.), Statistical Ecology. A primer on methods and computing. A Wiley-Interscience publication. 337 p.

Taylor, W. R. (1961). Aggregation variance and the mean. Nature, 189 (4766), 732-735.

Valdivia, A. y De la Guardia, E. (2004). Estructura de la comunidad de corales en el arrecife costero de Boca de Canasí, La Habana, Cuba. Rev. Invest. Mar., 25 (1), 15-22.

Varona, G., Caballero, H. y De la Guardia, E. (2004). Estructura ecológica de las comunidades de Octocorales de la costa oriental de Bahía de Cochinos, Cuba. Rev. Invest. Mar., 25 (3), 209-218.

Wulff, J. L. (1995). Effects of a hurricane on survival and orientation 
of large erect coral reef sponges.

Coral Reefs, 14, 55-61.

Zea, S. (2001). Patters of Sponges

(Porifera, Demospongiae) distribution in remote oceanic reef complexes of the Southwestern Caribbean. Rev. Acad. Coloma. Cien., XXV, 97, 579-592. 